\title{
Pancreatic INI1-deficient undifferentiated rhabdoid carcinoma achieves complete clinical response on gemcitabine and nab-paclitaxel following immediate progression on FOLFIRINOX: a case report
}

\author{
Daniel A. King ${ }^{1}$, Smruti Rahalkar ${ }^{1}$, David B. Bingham ${ }^{2}$, George A. Fisher ${ }^{1}$ \\ ${ }^{1}$ Department of Medicine, Stanford University, Stanford, CA, USA; ${ }^{2}$ Department of Pathology, Stanford University, Stanford, CA, USA \\ Correspondence to: Daniel A. King, George A. Fisher. Department of Medicine, Division of Oncology, Stanford University, 875 Blake Wilbur Drive, \\ Stanford, CA 94305, USA. Email: danking@stanford.edu; georgeaf@stanford.edu.
}

\begin{abstract}
Introduction: INI1-deficient undifferentiated rhabdoid carcinoma is a rare pancreatic carcinoma for which the optimal treatment is unknown. Pancreatic ductal adenocarcinoma, the most common histology of pancreas cancer, is treated with combination chemotherapy in the advanced setting, a strategy supported by strong evidence in well powered studies. In patients with excellent performance status, first-line treatment usually consists of the three-drug regimen FOLFIRINOX, with the combination of gemcitabine with nabpaclitaxel, typically less toxic than the three-drug regimen, reserved for second-line therapy. Given the lack of published reports describing treatment outcomes for patients with rare forms of pancreatic cancer, the same treatment approach used for pancreatic ductal adenocarcinoma is typically employed. Observation: This case describes a patient with metastatic pancreatic INI1-deficient undifferentiated rhabdoid carcinoma who was primarily resistant to FOLFIRINOX therapy but who then achieved an immediate, marked and sustained response to gemcitabine with nab-paclitaxel. Conclusion: Given the lack of data informing on optimal management of INI1-deficient pancreatic undifferentiated rhabdoid carcinoma, and the exceptional response achieved by gemcitabine with nab-paclitaxel, this case report highlights a surprising and potentially informative anecdote. Additional studies are needed to confirm responses observed in this report which when taken together may strongly influence first-line therapy choice for this rare malignancy. Given the difficult in acquiring sufficient numbers of these rare histologies in any one institution, multi-institution collaboration in studying outcomes of rare pancreatic malignancies is likely essential.
\end{abstract}

Keywords: INI1-deficient undifferentiated rhabdoid carcinoma; sarcomatoid undifferentiated carcinoma; pancreatic cancer; case report

Submitted Oct 31, 2020. Accepted for publication Jan 17, 2021.

doi: 10.21037 /jgo-20-478

View this article at: http://dx.doi.org/10.21037/jgo-20-478

\section{Introduction}

The optimal treatment of the rare malignancy INI1deficient undifferentiated rhabdoid carcinoma is not known. This report describes a case of INI1-deficient undifferentiated rhabdoid carcinoma that achieved an exceptional response to gemcitabine with paclitaxel despite immediate progression on FOLFIRINOX.

We present the following article in accordance with the CARE reporting checklist (available at: http://dx.doi. org/10.21037/jgo-20-478).

\section{Case presentation}

A 59-year-old woman presented to the emergency room in March 2019 with acute abdominal pain (Figure 1). She was in otherwise good health, and past medical history 


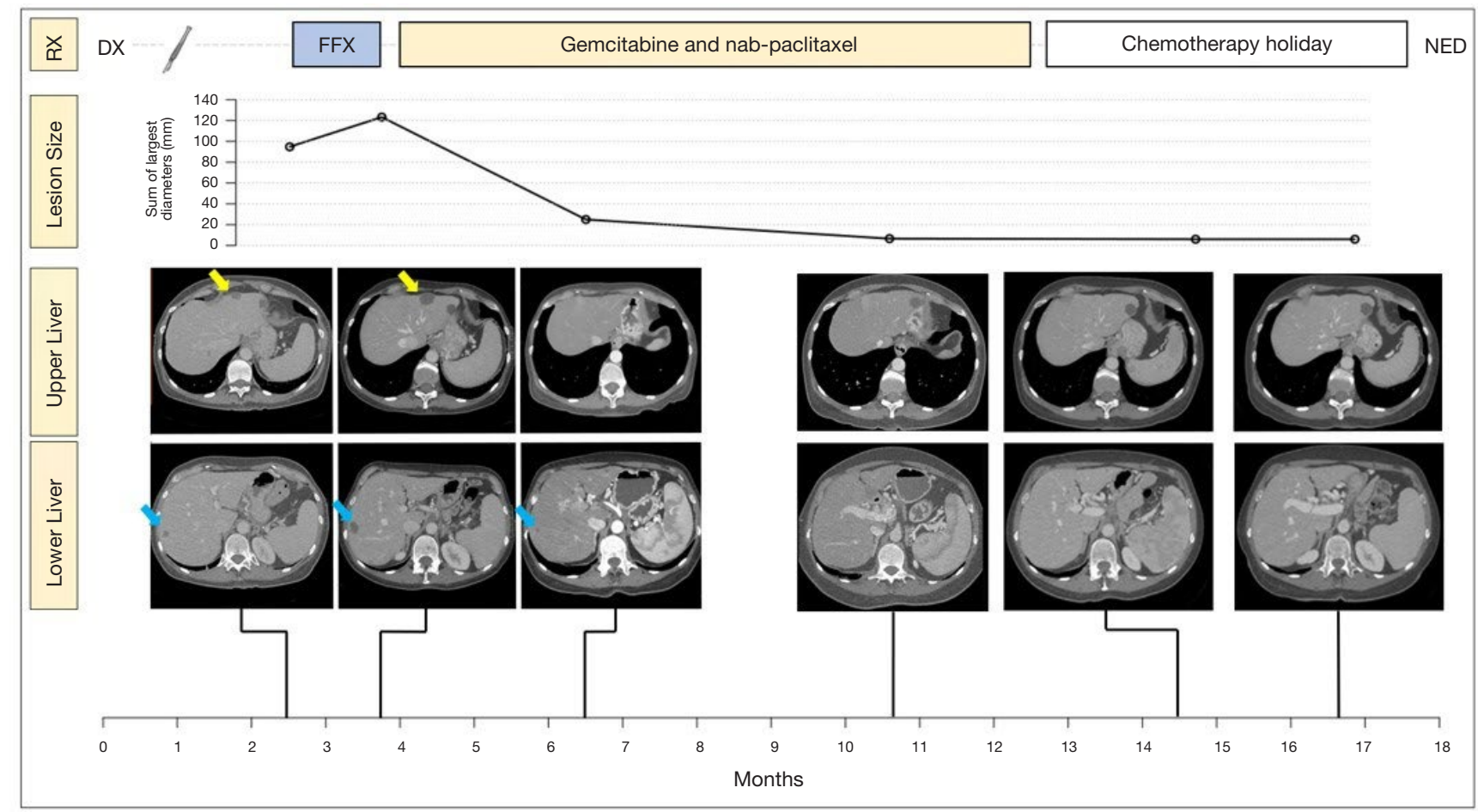

Figure 1 Treatment timeline. The $\mathrm{x}$-axis displays the clinical course, with rows signifying: treatments received (Rx), radiographic burden of disease measured by calculating the sum of the maximal tumor dimensions of each liver lesion (Lesions Size), and characteristic lesions displayed (Upper Liver; Lower Liver; yellow and blue arrows). Initiation of gemcitabine and nab-paclitaxel led to an immediate and sustained decrease in tumor burden.

consisted only of a chondrosarcoma of the cranium, diagnosed in 2013, which was resected, radiated, and remained in remission through surveillance. There was no significant family medical history. On presentation in 2019, CT demonstrated a mass with solid and cystic components in the tail of the pancreas with no evidence of metastatic disease. FDG-PET showed intense uptake (SUV 12) in the pancreatic mass and no evidence of metastases. An EUSguided FNA biopsy was highly suspicious for carcinoma. Both CEA and CA19-9 were within normal limits.

In April 2019, she underwent a robotic distal pancreatectomy with findings of a $1.6 \mathrm{~cm}$ invasive carcinoma with negative margins but with lymphovascular and perineural invasion and 1 of 35 nodes with malignant involvement. Surgical pathology review demonstrated sheets of monomorphic epithelioid cells with eosinophilic cytoplasm and a faint rhabdoid appearance but without glandular architecture, thus prompting consideration of a wide differential of non-adenocarcinoma neoplasms. Synaptophysin and chromogranin were negative, providing no support for neuroendocrine differentiation.
There was no expression of p63, lending no support for myoepithelial or squamous differentiation. ERG and CD34 were negative, providing no support for vascular differentiation. HMB45 negativity ruled out perivascular epithelioid cell tumor (PEComa). Lack of Sox-10/S100 and CD45RB expression, refuted metastatic melanoma or hematolymphoid differentiation, respectively. Given the patient's prior chondrosarcoma, that histology was compared to the pancreatic specimen, but the morphology was not similar. CK19 and CK7 were positive while CDX2 was negative, consistent with a pancreatic neoplasm. Immunohistochemical staining was broadly positive for pan-cytokeratin CKmix, consistent with epithelial differentiation. Stains for INI1 (SMARCB1) demonstrated a complete loss of nuclear expression in the neoplastic cells (Figure 2). Additional molecular studies showed intact mismatch repair proteins, PD-L1 expression with CPS of 20, and HER2 IHC was negative. Targeted tumor mutational profiling with the Stanford Tumor Actionable Mutation Panel identified a KRAS G12D mutation and variants of unknown significance in EPHA2 C376F and 

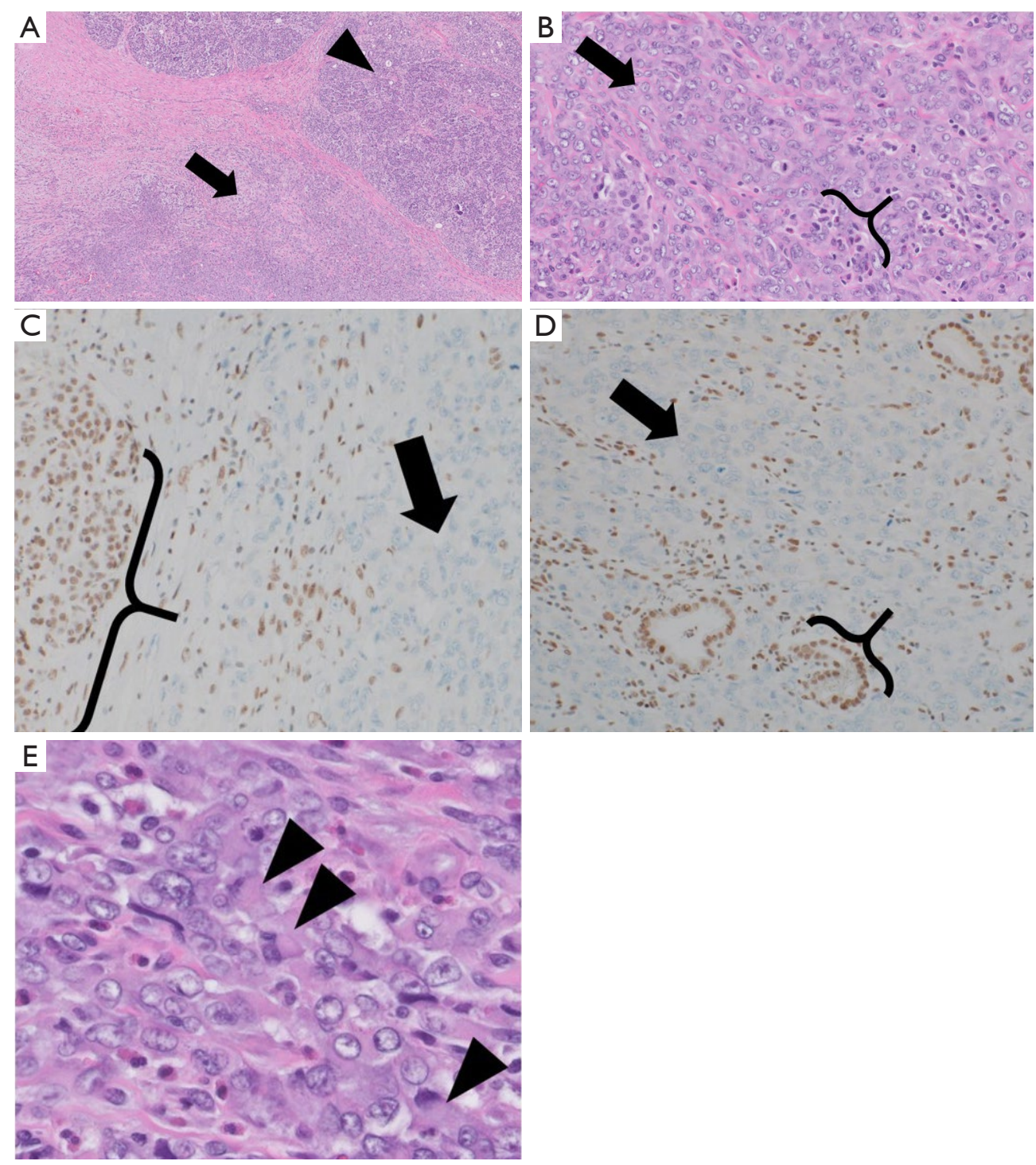

Figure 2 INI1-deficient tumor histology. (A) Lower power magnification (H\&E, 30x) showing: INI1-deficient tumor within pancreas (arrow) and surrounding background pancreatic tissue (arrowhead). (B) At higher power magnification (H\&E, 300x), the INI1-deficient tumor cells (arrow) are more readily visible, beside infiltrating lymphocytes (bracket). The predominant morphology was epithelioid. (C) INI1 immunostain at 300× magnification shows preserved INI1 staining in normal pancreatic cells, fibroblasts, and lymphocytes (bracket), which serve as internal positive controls, while the tumor cells (arrow) are not staining. (D) The INI1 immunostain at 300× magnification shows non-staining tumor cells (arrow) infiltrating pancreatic tissue with well stained pancreatic ductules and background fibroblasts and lymphocytes (bracket). (E) Lastly, arrowheads depict the pink cytoplasmic inclusions and displaced nuclei characteristic of rhaboid morphology.

a KDR L1095 frameshift, consistent with a low tumor mutational burden. Multigene testing for inherited mutations identified no abnormalities. The overall findings were that of a stage IIb (pT1c N1 M0) primary pancreatic INI1-deficient undifferentiated rhabdoid carcinoma.
Prior to a planned course of adjuvant chemotherapy, a CT in June 2019 demonstrated multiple [6-7] new liver metastases, the largest of which was $1.9 \mathrm{~cm}$. FNA of one of the lesions confirmed metastatic disease of similar histology. An attempt to grow organoids from the biopsy 
was unsuccessful.

She then received three doses of mFOLFIRINOX but due to progressive increase in right upper quadrant pain, a CT scan was ordered prior to a planned $4^{\text {th }}$ dose, which demonstrated an increase in size and number of liver metastases (Figure 1) warranting change in therapy.

In July of 2019, she was switched to gemcitabine $850 \mathrm{mg} / \mathrm{m}^{2}$ with nab-paclitaxel $100 \mathrm{mg} / \mathrm{m}^{2}$. Treatment on cycle 1 day 15 was held due to cytopenia. A short interval scan in August 2019, after receiving day 1 and day 8 of treatment, showed no new hepatic lesions and size stability of all known liver lesions. Treatment frequency was reduced to every other week dosing on account of the cytopenia.

Following two months of therapy, she had a dramatic clinical improvement, reporting that her abdominal pain had decreased to a level of 1-2 out of 10 and she had stopped taking all analgesics. She said that she felt as well as she did before her illness. Follow-up CT CAP in October 2019 showed marked reduction in the size of multiple hepatic metastases. She continued biweekly therapy. By November 2019, her abdominal pain had resolved completely. She developed peripheral neuropathy for which the nab-paclitaxel dose was reduced, and a rash, which was managed with prednisone. CT scan in December 2019 showed continued response, with all remaining liver disease subcentimeter in size. She started planning a long-distance vacation. By late January, CT no longer identified any lesions in the liver. In March of 2020, she began a chemotherapy holiday, and at scan and followup in August 2020, she remained without radiographic or clinical evidence of disease, and continues on surveillance off treatment at the time of submission.

All procedures performed in studies involving human participants were in accordance with the ethical standards of the institutional and/or national research committee(s) and with the Helsinki Declaration (as revised in 2013). Written informed consent was obtained from the patient.

\section{Discussion}

The histologic classification of undifferentiated carcinoma is complex. Undifferentiated rhabdoid carcinoma of the pancreas is quite rare with fewer than 100 cases reported in the literature (1). According to the latest WHO classification system, these tumors fall within the broader category of sarcomatoid undifferentiated carcinoma (2). Agaimy et al. have further subtyped undifferentiated rhabdoid carcinomas into two groups, a pleomorphic variant which tends to have KRAS alterations, and a monomorphic variant (such as the tumor discussed in this report) which tends to be INI1-deficient (1). Few data inform optimal treatment strategies for these very rare malignancies. A case report demonstrating complete response to a paclitaxel-containing regimen in pleomorphic variant undifferentiated pancreatic carcinoma was published in 2010 (3). SMARCB1/INI1-deficiency is a recently recognized molecular hallmark of renal medullary carcinoma (4), where paclitaxel-containing combinations have shown partial (5) and complete responses (6). To our knowledge, the work presented here is the first publication describing the clinical course and response to chemotherapy in a patient with metastatic INI1-deficient undifferentiated rhabdoid carcinoma.

Chromatin accessibility is a tightly regulated process and an integral determinant of gene expression. Disruption of chromatin remodeling can promote tumorigenesis $(7,8)$. The gene INI1, also known as SMARB1, for SWI/SNFrelated matrix-associated actin-dependent regulator of chromatin subfamily B member 1 , encodes for proteins involved in relieving repressive chromatin structures, thus acting as a tumor suppressor gene (9). Its carcinogenicity is in part linked to is dysregulation of cyclin-dependent kinase inhibitor p16, Wnt signaling, and E2F factors, among others (10). Following diagnosis in any age or organ, nearly all SMARCB1/INI1-deficient malignancies typically follow an aggressive clinical pattern and have a poor prognosis (1).

On presentation, the patient was 59 years old, with excellent performance status after her operation. Lacking specific guidelines to inform therapy selection in this rare histology, chemotherapy was chosen based on extrapolation for pancreatic ductal adenocarcinoma. Thus, firstline therapy with FOLFIRINOX was chosen, which is associated with a median overall survival of 11 months in patients with metastatic pancreatic adenocarcinoma (11). The cancer immediately progressed through first line therapy. Gemcitabine with nab-paclitaxel was chosen for second-line therapy, and the patient had an immediate response. The lack of long-term follow-up is a limitation of this case report. Nevertheless, the response thus far achieved was remarkable: two studies have explored the response rate of second-line gemcitabine with nab-paclitaxel in metastatic pancreatic ductal adenocarcinoma following FOLFIRINOX therapy: (I) in a study of 30 patients, including 11/30 (37\%) who had progressive disease on FOLFIRINOX, there were $0 / 30$ patients on gemcitabine with nab-paclitaxel who then achieved a complete response 
and only 4/30 (13\%) achieved a partial response (12); and (II) among 57 patients, including 16 of 57 (28\%) who had progressive disease on FOLFIRINOX, there were 0 of 57 patients achieving a complete response and only 22 of $57(39 \%)$ achieving a partial response (13). Thus, while primary refractoriness to FOLFIRINOX is not rare $(37 \%$, and $27 \%$ ), achieving a complete response to gemcitabine with nab-paclitaxel has not been previously reported and thus this case is exceptional.

The underlying mechanism explaining the complete clinical response to gemcitabine with nab-paclitaxel despite rapid progression on FOLFIRINOX is not clear. We speculate that the excellent response achieved with gemcitabine and nab-paclitaxel may be related to the different mechanism of action of the latter, which may better synergize with INI1 deficiency-mediated defects in chromatin remodeling. We note an established link between defects in chromatin remodeling and mitotic spindle function (14), suggesting a role for synergism or synthetic lethality when combining INI1 deficient neoplasms with microtubule inhibitors. Combining HDAC inhibitors with other therapeutics in pancreatic cancer is an area of active investigation (15), but has met with limited success. For example, trials in pancreatic ductal adenocarcinoma using HDAC inhibitors along with gemcitabine have largely been disappointing (8). It has been shown that HDAC inhibitors potentiate topoisomerase I-mediated DNA damage (16) and exploiting the rationale that $\mathrm{HDAC}$ inhibitors may sensitize tumors to topoisomerase inhibitors is an area of active study (17). As an alternative explanation, we note that while CPS score is not commonly used as a predictive biomarker in pancreatic cancer, the CPS score in this case was 20, suggesting a robust degree of immunogenicity of the tumor; perhaps this PD-L1 staining immune compartment primed an immune response for second-line therapy. Ultimately, a greater mechanistic understanding is needed to identify predictive biomarkers of response for this rare pancreatic neoplasm.

In conclusion, while the optimal treatment for INI1deficient undifferentiated rhabdoid carcinoma of the pancreas is not known, this clinical case report highlights an exceptional response to gemcitabine with nab-paclitaxel, despite initial failure of FOLFIRINOX, which may rightfully influence firstline treatment selection in this rare malignancy.

\section{Acknowledgments}

Funding: None.

\section{Footnote}

Reporting Checklist: The authors have completed the CARE reporting checklist. Available at: http://dx.doi.org/10.21037/ jgo-20-478

Conflicts of Interest: All authors have completed the ICMJE uniform disclosure form (available at: http://dx.doi. org/10.21037/jgo-20-478). George Fisher reports personal fees from Merck, Taiho, Roche, Terumo and Ipsen; and has served data safety monitoring boards for Astra Zeneca, Hutchison Pharma and Cytom X and Silenseed. The other authors have no conflict of interests to disclose.

Ethical Statement: The authors are accountable for all aspects of the work in ensuring that questions related to the accuracy or integrity of any part of the work are appropriately investigated and resolved. All procedures performed in studies involving human participants were in accordance with the ethical standards of the institutional and/or national research committee(s) and with the Helsinki Declaration (as revised in 2013). Written informed consent was obtained from the patient.

Open Access Statement: This is an Open Access article distributed in accordance with the Creative Commons Attribution-NonCommercial-NoDerivs 4.0 International License (CC BY-NC-ND 4.0), which permits the noncommercial replication and distribution of the article with the strict proviso that no changes or edits are made and the original work is properly cited (including links to both the formal publication through the relevant DOI and the license). See: https://creativecommons.org/licenses/by-nc-nd/4.0/.

\section{References}

1. Agaimy A, Haller F, Frohnauer J, et al. Pancreatic undifferentiated rhabdoid carcinoma: KRAS alterations and SMARCB1 expression status define two subtypes. Mod Pathol 2015;28:248-60.

2. Nagtegaal ID, Odze RD, Klimstra D, et al. The 2019 WHO classification of tumours of the digestive system. Histopathology 2020;76:182-8.

3. Wakatsuki T, Irisawa A, Imamura H, et al. Complete response of anaplastic pancreatic carcinoma to paclitaxel treatment selected by chemosensitivity testing. Int J Clin Oncol 2010;15:310-3.

4. Jia L, Carlo MI, Khan H, et al. Distinctive mechanisms underlie the loss of SMARCB1 protein expression in renal 
medullary carcinoma: morphologic and molecular analysis of 20 cases. Mod Pathol 2019;32:1329-43.

5. Gangireddy V gopala reddy, Liles GB, Sostre GD, et al. Response of metastatic renal medullary carcinoma to carboplatinum and paclitaxel chemotherapy. Clin Genitourin Cancer 2012;10:134-9.

6. Walsh A, Kelly DR, Vaid YN, et al. Complete response to carboplatin, gemcitabine, and paclitaxel in a patient with advanced metastatic renal medullary carcinoma. Pediatr Blood Cancer 2010;55:1217-20.

7. Wolffe AP. Chromatin remodeling: Why it is important in cancer. Oncogene 2001;20:2988-90.

8. Hessmann E, Johnsen SA, Siveke JT, et al. Epigenetic treatment of pancreatic cancer: Is there a therapeutic perspective on the horizon? Gut 2017;66:168-79.

9. Hollmann TJ, Hornick JL. INI1-deficient tumors: Diagnostic features and molecular genetics. Am J Surg Pathol 2011;35:e47-63.

10. Kalimuthu SN, Chetty R. Gene of the month: SMARCB1. J Clin Pathol 2016;69:484-9.

11. Conroy T, Desseigne F, Ychou M, et al. FOLFIRINOX

Cite this article as: King DA, Rahalkar S, Bingham DB, Fisher GA. Pancreatic INI1-deficient undifferentiated rhabdoid carcinoma achieves complete clinical response on gemcitabine and nab-paclitaxel following immediate progression on FOLFIRINOX: a case report. J Gastrointest Oncol 2021;12(2):874-879. doi: 10.21037/jgo-20-478 versus Gemcitabine for Metastatic Pancreatic Cancer. N Engl J Med 2011;364:1817-25.

12. Mita N, Iwashita T, Uemura S, et al. Second-Line Gemcitabine Plus Nab-Paclitaxel for Patients with Unresectable Advanced Pancreatic Cancer after First-Line FOLFIRINOX Failure. J Clin Med 2019;8:761.

13. Portal A, Pernot S, Tougeron D, et al. Nab-paclitaxel plus gemcitabine for metastatic pancreatic adenocarcinoma after Folfirinox failure: An AGEO prospective multicentre cohort. Br J Cancer 2015;113:989-95.

14. Giaccia AJ. A New Chromatin-Cytoskeleton Link in Cancer. Mol Cancer Res 2016;14:1173-5.

15. Baretti M, Ahuja N, Azad NS. Targeting the epigenome of pancreatic cancer for therapy: challenges and opportunities. Ann Pancreat Cancer 2019;2:18.

16. Thurn KT, Thomas S, Moore A, et al. Rational therapeutic combinations with histone deacetylase inhibitors for the treatment of cancer. Future Oncol 2011;7:263-83.

17. Seo YH. Dual Inhibitors Against Topoisomerases and Histone Deacetylases. J Cancer Prev 2015;20:85-91. 